\title{
L'exotisme synesthésique dans la poésie de Baudelaire $\left(^{*}\right)$
}

\author{
Nada S. Al-Nasser \\ Academic at Yarmouk University
}

\begin{abstract}
This study shed the light on the sensory homogeneity phenomenon " Synesthesia" by presenting the scientific definition for it, and applying it in the literary work of Baudelaire. This paper will present how this neurological and physiological phenomenon passed successfully to art domains, not just in theater and in music, but it went beyond to literary field.
\end{abstract}

In fact, Baudelaire was one of the first who believed in this phenomenon. He found it a rich source for inspiration, and a first step toward a modern literary revolution for the French poem. Therefore, the French poet created , by this theory, his own aesthetic theory" The Correspondences", which sees the human senses beyond its main function and aims to mix them, all this to arrive to idealism in expression and to pave the road toward symbolism which Consider Baudelaire one of its pioneers loyal.

However, the poet has a strange intuition in this area. He starts to study every things psychologically and to establish a network, on the one hand, between the reality and the symbol, and on other hand, between the past and the future to connect the poem to the immortality

What is Synesthesia? How did our poet develop this sensory phenomenon of physiological origin into a wonderful color adorned by the French poem and boasted through the ages? How did he benefit from the previous sensory theories in his work? And, finally, how did he treat this sensory phenomenon in his poetic works? We will try to answer to all of these questions with an amount of exceptional examples from literary production of Baudelaire.

${ }^{(*)}$ Bulletin of the Faculty of Arts Volume 79 Issue 8 October 2019 


\section{غرابة السينيستيزيا في شعر بودلير ناى صالح صالح الناصر}

أكاديمي في قسم اللغات الحديثة/ الأدب الفرنسي الدديث والمقارن/ جامعة اليرموك

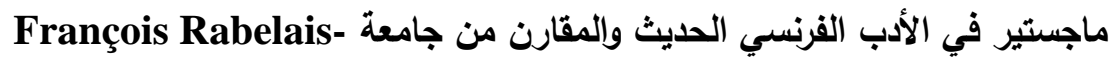

\section{Tours- La France}

يهدف هذا البحث إلى تسليط الضوء على ظاهرة السينيستيزيا أو ما تعرف بالترافق الحسي "La synesthésie"و ذللك بتقديم التعريف العلمي لها، و إسقاطه بالكلية على الإنتاج الأدبي للشاعر بودلير • سنتعرف في هذا المقال كيف تحولت هذه الظاهرة العصبية و المرتبطة بفسيولوجية أعضاء الإنسان إلى ظاهرة فنية لم تكتق قط بالدخول إلى المسرح أو الموسيقى أو حتى الرسم بل تعدت إلى الساحة الأدبية .

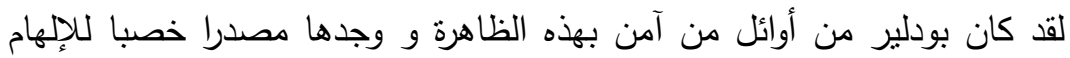

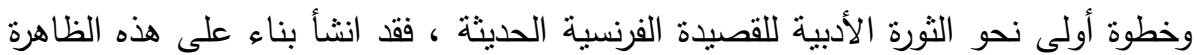

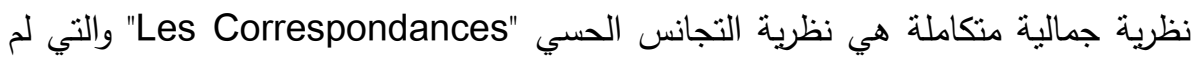
تعد ترى حواس الإنسان في إطار وظيفتها الأساسية بل تعدت إلى المزج بينها و تحقيق نيق

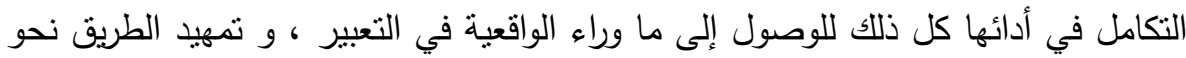

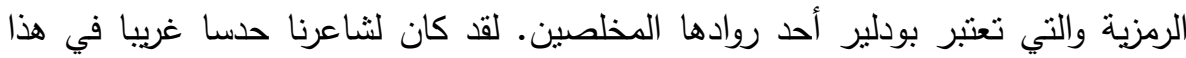

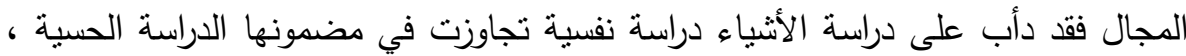
كل ذللك لإقامة شبكة تربط بين الحقيقة و الخيال، بين الواقع و الرمز، بين الماضي درابه

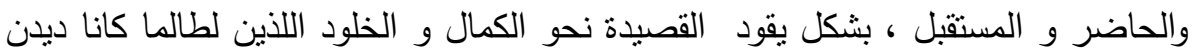

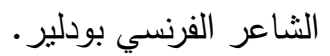
ما هي السينيسنيزيا ؟ وكيف حول شاعرنا هذه الظاهرة الحسية ذات الأصل

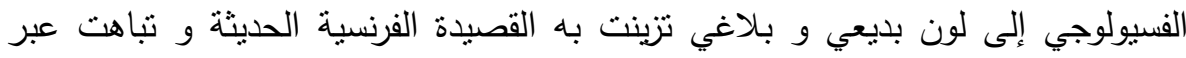

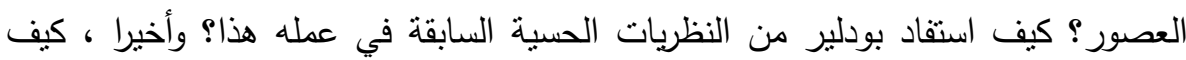

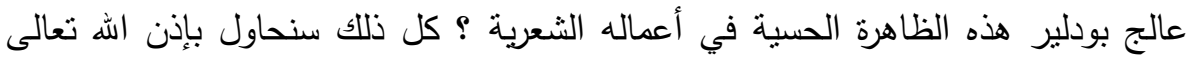
الإجابة عليه مستعينين بأمتلة متنوعة و مقتبسة من إرث بودلير الأدبي سائلين المولى العلي

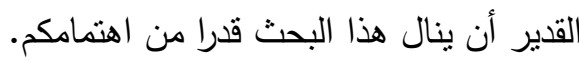




\section{Introduction}

Comme de longs échos qui de loin se confondent. Dans une ténébreuse et profonde unité, Vaste comme la nuit et comme la clarté, Les parfums, les couleurs et les sons se répondent (Baudelaire, 1869, p. 92).

Bien qu'elle soit un phénomène sensoriel d'origine neurologique qui concerne effectivement la science physiologique, la synesthésie devient plus en plus un sujet bien fréquent dans plusieurs domaines, tels que : en théâtre, en musique, en peinture et elle ne s'arrête pas là mais elle dépasse au champ littéraire. En fait, la synesthésie attire bien l'attention des hommes des lettres depuis longtemps, et plus particulièrement, depuis le $\mathrm{XIX}^{\mathrm{e}}$ siècle considéré comme le siècle de la modernité, de la bizarrerie et de la révolution en littérature. Autrement dit, c'est le siècle où la poésie de Baudelaire devient en tête des ouvrages littéraires pendant cette période grâce à son excellence en expression et à ses idées se trouvant exclusivement dans l'imaginaire baudelairien. C'est ainsi que notre but dans cet article c'est d'analyser le rôle de la synesthésie dans la poésie de Baudelaire et de mieux comprendre les raisons qui résident derrière sa rareté et son exception par rapport aux autres œuvres littéraires du $\mathrm{XIX}^{\mathrm{e}}$ siècle.

Tout d'abord, la synesthésie, venant du grec "syn" qui désigne l'ensemble et l'unité, tandis que " aesthesis" indique la perception, se définit ainsi que Hermann Parret évoque, il dit :

Qu'en est-il de la synesthésie? Dans sa définition la plus simple, la synesthésie est un phénomène d'association constante, chez un même sujet, d'impressions venant de domaines sensoriels différents. Sunaisthèsis, dans le langage philosophique grec, signifiait déjà l'action de percevoir une chose en même temps qu'une autre ; sensation ou perception simultanée". La synesthésie est souvent considérée comme étant d'origine 
pathologique, comme un trouble de la perception sensorielle dans lequel une sensation normale s'accompagne automatiquement d'une sensation complémentaire simultanée dans une régions du corps différente de celle où se produit l'excitation ou dans un domaine sensoriel différent (Parret, 2006, p. 62).

Cependant, la synesthésie est l'association arbitraire de deux ou de plusieurs sens. C'est une stimulation obligatoire et automatique qui se réalise simultanément entre deux ou plus de sensations, l'une superpose l'autre. Certes, cette alliance des sensations prend beaucoup de formes, telles que: la synesthésie "graphème-couleur" qui est considérée comme le type de la synesthésie le plus célèbre et qui s'appuie également sur le fait que les chiffres et les lettres évoquent les couleurs. D'ailleurs, il existe la synesthésie "synoptique" par laquelle les synesthèses voient des couleurs en écoutant de la musique, en voyant une image et même en respirant des odeurs. En plus, il y a de la synesthésie de personnification qui se cristallise autour de l'établissement des relations logiques entre les couleurs et les personnages. Pourtant, ce phénomène est un résultat d'arrivée des substances blanches dans le cerveau qui est, de sa part, responsable de faire les connexions entre les zones du Cortex et le transfert d'informations.

Quant à Baudelaire, il se trouve parmi les premiers à considérer la synesthésie comme :

Acte essentiellement mystique [....] comme processus révélateur des liens analogiques, conduisant de la multiplicité vers l'unité profonde de l'être, et dont la beauté est épiphanie dans le monde (Cigada, 2011, p. 29).

Aussi l'auteur des Fleurs du Mal est-il en proie à cette esthétique d'origine neurologique dans ses poèmes ce qui rend de lui effectivement le fondateur de l'école symboliste qui considère la synesthésie, se chargeant toujours de rendre visibles les choses invisibles, comme son principe initial sur lequel elle se fonde. 
Or, Baudelaire, le poète maudit et moderne, ne voit en ce phénomène sensoriel qu'une merveilleuse façon, d'une part, de trouver de nouveaux styles poétiques en projetant une lumière sur les craintes de la vie et sur les conditions humaines et d'autre part, d'ouvrir une autre voie vers la révolution littéraire dans la poésie française moderne.

Le statut de la synesthésie est ambivalent : elle est généralement réservée à un secteur particulier du discours littéraire, la poésie dite moderne, que la critique, par déférence et reconnaissance à l'égard de Baudelaire, fait commencer au milieu du XIX siècle. Dans cet esprit, le statut discursif de la synesthésie se rapproche de celui de la figure de rhétorique, dans la mesure où une certaine doxa entretient l'idée que le discours littéraire use et abuse de la rhétorique, tandis que le discours commun, si cette expression a un sens, s'en abstiendrait, et ce, malgré l'avertissement de Dumarsais ( Rioux, 2007, p. 221).

Dans le corpus baudelairien, aucune esthétique plus féconde que celle de la synesthésie. L'expérience stylistique, que ce soit création, que ce soit perception, dans les œuvres littéraires baudelairiennes est toujours sensorielle : la couleur symbolise la musique, la musique s'accorde harmoniquement avec la poésie et la poésie n'est que des sens divers empruntés à la nature.

Ce phénomène apparait effectivement en richesse au cours de la production littéraire de Baudelaire, à titre d'exemples : L'exposition universelle de 1855, Edgar Poe, sa vie et ses œuvres de 1856 , où Baudelaire indique la relation entre : la littérature, le théâtre, la musique et la peinture ; Le Poème de hachisch ; le sonnet de 1858ou encore l'article de Wagner publié en 1861. Cependant, l'idée de ce phénomène sensoriel se cristallise premièrement autour de la couleur dans le Salon de 1846, plus particulièrement, dans le troisième chapitre intitulé De la couleur où Baudelaire tente de formuler une 
nouvelle théorie de la couleur en peinture en renvoyant au Kreisleriana de Théodore Hoffmann :

Je me rappelle un passage d'Hoffmann qui exprime parfaitement mon idée, et qui plaira à tous ceux qui aiment sincèrement la nature: "Ce n'est pas seulement en rêve, et dans le léger délire qui précède le sommeil, c'est encore éveillé, lorsque j'entends de la musique, que je trouve une analogie et une réunion intime entre les couleurs, les sons et les parfums. Il me semble que toutes ces choses ont été engendrées par un même rayon de lumière, et qu'elles doivent se réunir dans un merveilleux concert. L'odeur des soucis bruns et rouges produit surtout un effet magique sur ma personne ( Baudelaire, 1975, p. 425-426).

Toutefois, il ne faut pas oublier qu'il y a des hommes de lettres $\mathrm{du} \mathrm{XX}^{\mathrm{e}}$ siècle qui intègrent la synesthésie dans leurs œuvres littéraires, tels que : Kandinsky ${ }^{1}$ dans sa pièce Der gelbe Klang, c'est à dire, le son jaune. Parallèlement, il existe beaucoup d'artistes qui rendent compte dans leur art de cette esthétique, tels que : le rocker Tom Yorke; le pianiste Franz Liszt ou encore le jazzman Duke Ellington.

Quelle est la synesthésie baudelairienne? Comment Baudelaire peut-il changer ce phénomène sensoriel en une esthétique littéraire bien célèbre? De quelles théories concernant les sensations Baudelaire profite-il de sorte qu'il puisse les appliquer aux lettres? Comment la vision baudelairienne traite-elle la question de la synesthésie dans ses œuvres littéraires, plus particulièrement, dans Les Fleurs du Mal et Le Spleen de Paris? Et, finalement, où pouvonsnous trouver des exemples soutenant la présence de la synesthésie dans le corpus des ouvrages littéraires de Baudelaire?

1 Peintre, théoricien, dramaturge et graveur russe( 1866-1944). Il est considéré comme le fondateur de l'art abstrait grâce à ces grands efforts dans ce domaine notamment la question d'établir une relation entre la musique et les couleurs. 


\section{Baudelaire et la synesthésie littéraire : entre la théorie et la pratique}

En disant, tout d'abord, que la synesthésie, inspirée d'une théorie chrétienne et médiévale du XIII ${ }^{\mathrm{e}}$ siècle, s'articule autour des éléments du microcosme et ceux du macrocosme naturel, Baudelaire en profite en indiquant qu'il y a une sorte d'alliance entre le sens humain et l'état métaphysique. Pourtant, il déploie de grands efforts en vue de faire des relations entre les deux mondes : concret et abstrait. L'auteur des Fleurs sait bien comment déchiffrer les sens des analogies en dépassant le monde des perceptions à ce lui des idées.

Cependant, Baudelaire reprend à son compte les travaux de certains théoriciens tels que Swedenborg et Lavater, et ceux des romantiques allemands comme Hoffmann et Novalis qui ont leur intuition particulière de la synesthésie; ainsi l'auteur peut-il développer visiblement l'idée invisible. D'ailleurs, il peut avec succès changer la synesthésie en une esthétique littéraire bien exotique qui devient plus tard le manifeste d'inspiration chez les symbolistes.

D'ailleurs Swedenborg, qui possédait une âme bien plus grande, nous avait déjà enseigné que le ciel est un très grand homme, que tout, forme, mouvement, nombre, couleur, parfum, dans le spirituel comme dans le naturel, est significatif, réciproque, converse correspondant. Lavater, limitant au visage de l'homme la démonstration de l'universelle vérité, nous avait traduit le sens spirituel du contour, de la forme, de la dimension ( Arnold, 1972, p. 147).

Tout le visible adhère à de l'invisible, tout l'audible à de l'inaudible, tout le sensible à du non sensible. Sans doute tout ce qui peut être pensé adhère-t-il de même à ce qui ne peut pas être pensé ( Kanters, 1950, p. 365).

Comme Hoffmann, Swedenborg, Fourier, Eliphas Levi, 
Balzac, Saint- Martin et Nerval, la synesthésie ne présente, d'après Baudelaire, qu'une interprétation philosophique et un phénomène psychologique qui tente de toucher l'infini et d'arriver à un univers unitaire et harmonieux en se servant d'un langage rhétorique bien maitrisé et capable d'analyser les relations objectives et analogiques dans l'univers entier. Aussi la synesthésie baudelairienne vise-t-elle à lire le poème comme une traduction du verbe absolu; ainsi Baudelaire affirme -t-il dans sa lettre à Alphonse Toussenel le 21 janvier 1856, il dit:

L'homme raisonnable n'a pas attendu que Fourier vînt sur la terre pour comprendre que la Nature est un verbe, une allégorie, un moule, un repoussé, si vous voulez. Nous savons cela, et ce n'est pas par Fourier que nous le savons;- nous le savons par nous-mêmes et par les poètes (Baudelaire, 1947,p. 370).

Alors, comme nous avons déjà souligné, la synesthésie baudelairienne est une opération anthropologique et une tâche esthétique précise. Sans doute l'art se charge-t-il de créer l'harmonie qui unifie l'objectivité à l'état métaphysique. Tout cela vient grâce à l'imagination, ainsi Baudelaire déclare-t-il dans son Salon de 1859(qui apparait, non par hasard, sous titre La Reine des facultés). Donc, l'imagination est celle :

qui a enseigné à l'homme le sens moral de la couleur, du conteur, du son et du parfum. Elle a créé, au commencement du monde, l'analogie et la métaphore ( audelaire, 1973, p. 532).

Or, pour l'auteur des Fleurs, les relations objectives parmi les sens correspondent à la nature. Baudelaire comprend bien cette réalité en essayant de la montrer d'une manière abstraire dans sa poésie. Cette conception esthétique et éthique présente en force dans l'article sur Wagner que Baudelaire a publié en 1861 à l'occasion de l'apparition de Tannhäuser à Paris :

Le lecteur sait quel but nous 
poursuivons : démontrer que la véritable musique suggère des idées analogues dans des cerveaux différents. D'ailleurs, il ne serait pas ridicule ici de raisonner à priori, sans analyse et sans comparaisons[...] c'est que le son ne pût pas suggérer la couleur, que les couleurs ne pussent pas donner l'idée d'une mélodie, et que le son et la couleur fussent impropres à traduire des idées (Baudelaire, 1980,p. 852).

En outre, il semble que le Salon de 1846 n'est qu'une traduction et qu'une analyse des tableaux de Delacroix où Baudelaire tente d'établir une relation entre la synesthésie et la peinture. En un mot, d'après Baudelaire, la production artistique de Delacroix est une ressource riche et féconde en inspiration et en imagination sur lesquelles la synesthésie baudelairienne s'est fondée :

Edgar Poe dit, je ne sais plus où, que le résultat de l'opium pour les sens est de revêtir la nature entière d'un intérêt surnaturel qui donne à chaque objet un sens plus profond, plus volontaire, plus despotique. [.....]. Et bien, la peinture de Delacroix me parait la traduction de ces beaux jours d'esprit. Elle est revêtue d'intensité et sa splendeur est privilégiée. Comme la nature perçue par des nerfs ultra-sensibles, elle révèle le surnaturalisme (Leakey, 1969, p. 181).

\section{La représentation de la synesthésie dans la poésie de Baudelaire.}

Sachons que la tâche baudelairienne fondée sur la mutation d'un phénomène neurologique à un autre phénomène littéraire n'a rien de simple, et que les efforts de Baudelaire dans ce domaine sont bien marqués comme nous allons le voir à travers certains exemples 
empruntés à l'héritage littéraire de Baudelaire. Autrement dit, la synesthésie n'est qu'une véritable bascule qui a réussi à faire une révolution radicale dans l'esthétique utilisée dans la littérature française.

Le premier exemple c'est le poème en prose L'invitation au voyage. Nous constatons ici un exemple exceptionnel s'appuyant sur la réunification et sur la fusion d'aspects différents qui travaillent ensemble d'une manière harmonique dans le but d'arriver à l'unité mystique de l'esthétique. Dans ce poème en prose de Baudelaire, nous touchons deux réalités objectives : un paysage splendide et une vieille amie, un pays idéal que le poète veut bien visiter avec son amie intime. En d'autres termes, nous trouvons un exemple où l'auteur révèle d'une manière minutieuse son intégration de la psychologie dans la technique rhétorique de l'association thématique :

Il est un pays superbe, un pays de Cocagne, dit-on, que je rêve de visiter avec une vieille amie. Pays singulier, noyé dans les brumes de notre Nord ( Baudelaire, 1968,p. 26).

Le texte poétique se poursuit, en richesse, la description de ce pays idéal et parfait en faisant une comparaison entre son amoureuse et ce paysage naturel : les deux fascinent le poète et les deux sont mystiques pour lui :

" Un vrai pays de Cocagne, où tout est beau, riche, tranquille, honnête $[\ldots]$; où tout vous ressemble mon cher ange. Tu connais cette maladie fiévreuse qui s'empare de nous dans les froides misères, cette nostalgie du pays qu'on ignore, cette angoisse de la curiosité ? Il est une contrée qui te ressemble, où tout est beau, riche, tranquille et honnête [...]. C'est là qu'il faut vivre, c'est là qu'il faut aller mourir"( Baudelaire, 1968,p. 26).

Pour Les Paradis artificiels, celui- ci ne parait qu'un essai de présenter certaines hallucinations causées par le hachisch. En ce cas là, il y a une déclaration du moi dans le texte poétique qui s'articule autour de la conscience lucide et de la volonté créatrice de l'auteur :

$$
\text { C'est en effet à cette période de }
$$
l'ivresse que se manifeste une finesse 
nouvelle, [...] Pour mieux comprendre ce bouillonnement d'imagination, cette maturation du rêve et cet enfantement poétique auquel est condamné un cerveau intoxiqué par le hachisch, je raconterai encore mon anecdote (Leakey, 1969, p. 207).

Remarquons également dans ce poème un autre exemple de l'unité entre l'homme et la nature "passage d'âme". A travers ce texte, il $\mathrm{y}$ a une alliance entre la présence humaine et le paysage. Ces derniers s'unissent harmoniquement de façon à arriver à l'imagination d'un lieu parfait et idéal.

Quant au poème les Correspondances, nous trouvons que les sons sont bien colorés et les parfums font avec eux une belle harmonie et une merveilleuse mélodie de musique: "Les parfums, les couleurs et les sons se répondent," (Baudelaire, 1869, p. 92). En effet, ce vers précédent révèle que la poésie de Baudelaire se charge d'unifier les choses que le réel sépare en donnant à chacune d'elles un sens verbal à la lumière du même texte. Les parfums, les couleurs et les sons se correspondent pour arriver à l'idéal que Baudelaire, le poète romantique, cherche.

En réalité, notre poète ne peut pas seulement créer cette théorie appelée " Les Correspondances" en faisant l'échange agréable des éléments sensoriels mais il affirme la coexistence différente de plusieurs états reliés aux sensations, mélangés et présentés simultanément, ce que Baudelaire tente de montrer dans sa fortune littéraire, telle que:

Vous êtes un beau ciel d'automne, clair et rose! ( Baudelaire, 1869, p. 171)

(Causerie v1)

Voici venir les temps où vibrant sur sa tige

Chaque fleur s'évapore ainsi qu'un encensoir ;

Les sons et les parfums tournent dans l'air du soir ; 
Valse mélancolique et langoureux vertige!

( Baudelaire, 1869, p. 155) (Harmonie du soir v1-4)

"Comme l'azur du ciel, les oiseaux et les

fleurs,

Ses parfums, ses chansons et ses douces chaleurs!( Baudelaire, 1869, p. 93)

(J'aime le souvenir de ces époques nues $v$ 39-40)

Je suis un cimetière abhorré de la lune,

Où comme des remords se traînent de longs vers

Qui s'acharnent toujours sur mes morts les plus chers (Baudelaire, 1869, p. 199).

( J'ai plus de souvenirs que si j'avais mille ans $v$ 7-9)

Et des habits, mousseline ou velours,

Tout imprégnés de sa jeunesse pure,

Se dégageait un parfum de fourrure.

Baudelaire, 1869, p. 141) ( Parfum v 9-11)

Car je serai plongé dans cette volupté

D'évoquer le Printemps avec ma volonté,

De tirer un soleil de mon cour, et de faire

De mes pensers brûlants une tiède

atmosphère ( Baudelaire, 1869, p. 250).

(Paysage v23-26)

Parmi toutes les belles choses

Dont est fait son enchantement,

Parmi les objets noirs ou roses

Qui composent son corps charmant, 

$[\ldots$.
Ô métamorphose mystique
De tous mes sens fondus en un!
Son haleine fait la musique,
Comme sa voix fait le parfum! (
Baudelaire, 1869, p. 192) (La Musique v
$5-8 / 21-24)$

En lisant attentivement les exemples suivants, nous pouvons trouver que la synesthésie est, en effet, une transcendance parfaite des éléments naturels bien divisés. Baudelaire les rend comme un miroir fidèle qui reflète bien ses émotions.

Au pays parfumé que le soleil caresse,

J'ai connu, sous un dais d'arbres tout empourprés

Et de palmiers d'où pleut sur les yeux la paresse,

Une dame créole aux charmes ignorés (Baudelaire, 1869, p. 183).( À une dame créole v 1-4)

Chaque fleur s'évapore ainsi qu'un encensoir ;

Le violon frémit comme un cour qu'on afflige;

Valse mélancolique et langoureux vertige!

Le ciel est triste et beau comme un grand reposoir (

Baudelaire, 1869, p. 155).( Harmonie du soir v 4-8)

Au-dessus des étangs, au-dessus des vallées,

Des montagnes, des bois, des nuages, des mers,

Par delà le soleil, par delà les éthers,

Par delà les confins des sphères étoilées,

Mon esprit, tu te meus avec agilité,

Et, comme un bon nageur qui se pâme dans l'onde,

Tu sillonnes gaiement l'immensité profonde

Avec une indicible et mâle volupté (Baudelaire, 1869, p. 90).(Elévation v 1-8) 
Ô Lune qu'adoraient discrètement nos pères, Du haut des pays bleus où, radieux sérail, Les astres vont se suivre en pimpant attirail, Ma vieille Cynthia, lampe de nos repaires, ( Baudelaire, 1869, p. 254) ( La lune offensée v 1-4)

Sois sage, ô ma Douleur, et tiens-toi plus tranquille. Tu réclamais le Soir; il descend; le voici: Une atmosphère obscure enveloppe la ville, Aux uns portant la paix, aux autres le souci.

Pendant que des mortels la multitude vile, Sous le fouet du Plaisir, ce bourreau sans merci, Va cueillir des remords dans la fête servile, Ma douleur, donne-moi la main ; viens par ici, ( Baudelaire, 1869, p. 239) ( Recueillement v 1-8)

Nous pouvons encore trouver cette technique d'écriture dans d'autres textes de Baudelaire, comme dans le magnifique Jet d'eau. En fait, ce poème se divise en 3 strophes, dans la première strophe, nous trouvons deux images totalement différentes et séparées : l'image de l'amoureuse et le jet de l'eau :

Tes beaux yeux sont las, pauvre amante!

Reste longtemps, sans les rouvrir,

Dans cette pose nonchalante

Où t'a surprise le plaisir.

Dans la cour le jet d'eau qui jase,

Et ne se tait ni nuit ni jour,

Entretient doucement l'extase

Où ce soir m'a plongé l'amour ( Baudelaire, 1869, p. 231). (Jet d'eau v1-8)

Dans la deuxième strophe, nous constatons la dualité des deux images : l'image de l'eau qui monte vers le haut et tombe immédiatement par terre, et l'image de l'amante qui s'élance dans l'amour et retombe ensuite très fatiguée et épuisée :

Ainsi ton âme qu'incendie

L'éclair brûlant des voluptés 
S'élance, rapide et hardie,

Vers les vastes cieux enchantés.

Puis elle s'épanche, mourante,

En un flot de triste langueur,

Qui par une invisible pente

Descend jusqu'au fond de mon cour (Baudelaire, 1869, p. 231). ( v 15-22)

Dans la troisième strophe, les sentiments de la mélancolie et de la tristesse dépassent le soi de l'auteur au paysage naturel. En un mot, le poète fait une analogie reliant son état d'âme à la nature qui l'entoure, comme nous l'avons souligné avant :

$\hat{O}$ toi, que la nuit rend si belle,

Qu'il m'est doux, penché vers tes seins,

D'écouter la plainte éternelle

Qui sanglote dans les bassins!

Lune, eau sonore, nuit bénie,

Arbres qui frissonnez autour,

Votre pure mélancolie

Est le miroir de mon amour ( Baudelaire, 1869, p.

232). ( v 29-36)

Après avoir lu certains exemples empruntés à la bibliothèque littéraire de Baudelaire, nous trouvons que la synesthésie baudelairienne est bien variée et vouée à l'harmonie. Parfois, nous la trouvons cosmique, ex : Tout entière, et d'autres fois, nous la voyons sensuelle, ex: Parfum exotique ou encore La chevelure. De toute façon, la femme dans le texte de Baudelaire reste le moyen par lequel le poète arrive à l'infini et à la perfection. A titre d'exemple : $L a$ chevelure, le poème de 1859 , dans lequel le poète se charge de considérer la synesthésie comme d'un inébriant qui conduit à l'ivresse de la création (Brombert, 1974, p. 54-63). C'est ainsi que Baudelaire dans ce poème ne cesse de toujours intégrer différentes perceptions sensorielles en en faisant, d'une manière harmonique, une merveilleuse chanson et une grandiose symphonie où la femme apparait comme l'instrument duquel le poète joue afin de créer un orchestre très rare et très sensible qui touche au fond du cœur de l'homme.

En fait, dans ce poème en vers, nous touchons un réseau 
synesthésique qui dirige la reconstitution textuelle du poème aux odeurs (ex : Ô parfum chargé de nonchaloir !"), au désir, ex :" Extase ! Pour peupler ce soir l'alcôve obscure", " amoureuse d'ivresse" et "Afin qu'à mon désir tu ne sois jamais sourde!") et au prolongement de l'espace et du temps (ex : "Des souvenirs dormant", " La langoureuse Asie "). Nous constatons d'ailleurs l'alliance des deux sens : tactile et visuel (ex : "Cheveux bleus"). Nous trouvons également l'association des trois sensations à la fois : l'odorat, l'audition et la vision ( ex : "À grands flots le parfum, le son et la couleur"). En conséquence, la fusion des sensations dans ce texte poétique reflète bien l'alliance avec toutes les parts de l'être entier, autrement dit, la synesthésie de Baudelaire est une métamorphose qui révèle son état spirituel et psychologique, comme nous l'avons déjà indiqué dans la troisième strophe du poème Le jet d'eau:

Ô toison, moutonnant jusque sur l'encolure!

Ô boucles! Ô parfum chargé de nonchaloir!

Extase! Pour peupler ce soir l'alcôve obscure

Des souvenirs dormant dans cette chevelure,

Je la veux agiter dans l'air comme un

mouchoir!

La langoureuse Asie et la brûlante Afrique,

Tout un monde lointain, absent, presque

défunt,

Vit dans tes profondeurs, forêt aromatique!

Comme d'autres esprits voguent sur la musique,

Le mien, ô mon amour! nage sur ton parfum.

J'irai là-bas où l'arbre et l'homme, pleins de sève,

Se pâment longuement sous l'ardeur des climats;

Fortes tresses, soyez la houle qui m'enlève!

Tu contiens, mer d'ébène, un éblouissant rêve

De voiles, de rameurs, de flammes et de mâts:

Un port retentissant où mon âme peut boire

À grands flots le parfum, le son et la couleur

Où les vaisseaux, glissant dans l'or et dans la moire

Ouvrent leurs vastes bras pour embrasser la 
gloire

D'un ciel pur où frémit l'éternelle chaleur.

Je plongerai ma tête amoureuse d'ivresse

Dans ce noir océan où l'autre est enfermé ;

Et mon esprit subtil que le roulis caresse

Saura vous retrouver, ô féconde paresse,

Infinis bercements du loisir embaumé!

Cheveux bleus, pavillon de ténèbres tendues

Vous me rendez l'azur du ciel immense et rond

Sur les bords duvetés de vos mèches tordues

Je m'enivre ardemment des senteurs confondues

De l'huile de coco, du musc et du goudron.

Longtemps! Toujours! ma main dans ta crinière lourde

Sèmera le rubis, la perle et le saphir,

Afin qu'à mon désir tu ne sois jamais sourde!

N'es-tu pas l'oasis où je rêve, et la gourde

Où je hume à longs traits le vin du souvenir?

(Baudelaire, 1869, p. 119).

En retournant aux Petits poèmes en prose, dits aussi Le spleen de Paris, nous remarquons bien que la synesthésie est un réseau qui s'est fondé sur la rhétorique métronomique et métaphorique à la fois. Cette nouvelle architecture textuelle est considérée comme une autre véritable révolution dans l'esthétique baudelairienne. C'est l'essor d'un style qui était réservé par l'harmonie métaphysique de la synesthésie à un autre type personnel et subjectif du réel. C'est ainsi que nous ne trouvons plus les sens fusionnels mais contigüs.

Néanmoins, le poème en prose Un hémisphère dans la chevelure correspond à celui en vers La chevelure : le premier se prolonge en 7 paragraphes tandis que le deuxième comprend 7 strophes, et le premier analyse les mêmes perceptions que le deuxième explique en répétant les mêmes idées, telles que: "nage sur ton parfum" et "Mon âme voyage sur le parfum"; " Des souvenirs dormant" et " que je mange des souvenirs"; " Longtemps !" et " retrouve les langueurs des longues heures passées" ; "un mouchoir odorant " et "comme un mouchoir!". Cependant, nous trouvons que le 
poème en prose Un hémisphère dans la chevelure est très riche en synesthésie, telle que : l'audition visuelle ( ex: "j'entends dans tes cheveux !"); l'association entre le goût et l'adorat (ex : "je respire l'odeur du tabac mêlé à l'opium et au sucre" ) et le désir et l'audition (ex: " Afin qu'à mon désir tu ne sois jamais sourde!").

Laisse-moi respirer longtemps, longtemps, l'odeur de tes cheveux, y plonger tout mon visage, comme un homme altéré dans l'eau d'une source, et les agiter avec ma main comme un mouchoir odorant, pour secouer des souvenirs dans l'air.

Si tu pouvais savoir tout ce que je vois! tout ce que je sens! tout ce que j'entends dans tes cheveux! Mon âme voyage sur le parfum comme l'âme des autres hommes sur la musique.

Tes cheveux contiennent tout un rêve, plein de voilures et de mâtures; ils contiennent de grandes mers dont les moussons me portent vers de charmants climats, où l'espace est plus bleu et plus profond, où l'atmosphère est parfumée par les fruits, par les feuilles et par la peau humaine.

Dans l'océan de ta chevelure, j'entrevois un port fourmillant de chants mélancoliques, d'hommes vigoureux de toutes nations et de navires de toutes formes découpant leurs architectures fines et compliquées sur un ciel immense où se prélasse l'éternelle chaleur.

Dans les caresses de ta chevelure, je retrouve les langueurs des longues heures passées sur un divan, dans la chambre d'un beau navire, bercées par le roulis imperceptible du port, entre les pots de fleurs et les gargoulettes rafraîchissantes. 
Dans l'ardent foyer de ta chevelure, je respire l'odeur du tabac mêlé à l'opium et au sucre; dans la nuit de ta chevelure, je vois resplendir l'infini de l'azur tropical; sur les rivages duvetés de ta chevelure je m'enivre des odeurs combinées $d u$ goudron, du musc et de l'huile de coco.

Laisse-moi mordre longtemps tes tresses lourdes et noires. Quand je mordille tes cheveux élastiques et rebelles, il me semble que je mange des souvenirs ( Baudelaire, 1975, p. 300-301).

\section{Conclusion}

Il ressort de tout ce que nous venons de dire que la synesthésie est un mot, créé du symbolisme, qui trouve ses racines anciennes dans le dictionnaire français qui possédait antérieurement l'adjectif "synesthésique". En fait, ce dernier désigne tout simplement un concept physiologique qui se cristallise autour de la question de la stimulation involontaire des sensations, autrement dit, la double sensation.

En outre, au XVIII ${ }^{\mathrm{e}}$ siècle, un jésuite, le $\mathrm{P}$. Castel a construit un clavecin oculaire sur lequel les sons se présentent en couleurs et grâce à lui les sourds peuvent entendre la musique. Ajoutons l'invention de Braille, qui est fondée sur la théorie de la vision tactile et qui aide heureusement les aveugles à lire.

Baudelaire, poète moderne et sensible, est parmi les premiers à se servir de la synesthésie dans la production littéraire et à appliquer certaines théories concernant ce domaine telles que : la vision sonore et l'audition colorée de la poésie ce qui conduit effectivement l'auteur des Fleurs à créer sa théorie esthétique qui s'appelle "Les Correspondances".

D'après Baudelaire, il ne s'agit pas simplement de trouver un monde poétique plein de liens analogiques. Les Fleurs du Mal, recueil baudelairien publié la première fois en 1857, Le Spleen de Paris de 1869 et les autres exemples que nous avons déjà mis en valeur au 
cours de cette recherche ne sont qu'un champ fécond de la fusion des sensations où le sens humain est en effet un instrument de la perception et des prémices de la pensée métaphysique sur lequel le symbole se fonde.

Grâce à tout ce qui précède, nous pouvons dire alors qu'en utilisant la synesthésie, Baudelaire peut avec succès écrire une poésie éternelle et capable de se graver dans les cœurs et dans les mémoires de ceux qui l'entendent en intégrant cette esthétique d'origine sensorielle. 


\section{Bibliographie sélective:}

Arnold, Paul. Esotérisme de Baudelaire, J.Vrin, Paris, 1972.

Baudelaire, Charles. Les Fleurs du Mal. Levy frères éditeurs. Paris, 1869.

Baudelaire, Charles, Crépet, Jaques. Les ceuvres complètes de Charles Baudelaire, Conard, Paris, 1947.

Baudelaire, Charles. Petits poèmes en prose, edited introduction and notes by Melvin Zimmerman, 1968.

Baudelaire, Charles. Les æeuvres complètes, Gallimard, Paris, 1973.

Baudelaire, Charles. Les æeuvres complètes, Gallimard, Paris, 1975.

Baudelaire, Charles. Les auvres complètes, Laffont, Paris, 1980.

Brombert, Victor. The will to ecstasy: Example of Baudelaire" Chevelure", in Yale French studies, n. 50, 1974.

Ciada, Sergio. Etudes sur le symbolisme, EDUCatt, Milan, 2011.

Hérbert, Louix. Le plaisir des sens: euphories et dysphories des signes, presses de l'université de Laval, Laval, 2007.

Kanters, Robert. Anthologie littéraire de l'occultisme, René Julliard, Paris , 1950.

Leakey, F.W. Baudelaire and Nature, Manchester university press, Manchester, 1969.

Parret, Herman. Essais sémio-esthétiques, presses de l'université de Limoges, Limoges, 2006. 